\title{
A nanomechanical device based on light-driven proton pumps
}

\author{
Quan Ren $^{1}$, Ya-Pu Zhao ${ }^{1,3}$, Li Han ${ }^{2}$ and Hui-Bin Zhao ${ }^{2}$ \\ ${ }^{1}$ State Key Laboratory of Nonlinear Mechanics (LNM), Institute of Mechanics, \\ Chinese Academy of Sciences, Beijing 100080, People's Republic of China \\ ${ }^{2}$ Institute of Electrical Engineering, Chinese Academy of Sciences, Beijing 100080, \\ People's Republic of China \\ E-mail: yzhao@lnm.imech.ac.cn (Y-P Zhao)
}

Received 11 September 2005, in final form 16 December 2005

Published 3 March 2006

Online at stacks.iop.org/Nano/17/1778

\begin{abstract}
In this paper, a hybrid device based on a microcantilever interfaced with bacteriorhodopsin (bR) is constructed. The microcantilever, on which the highly oriented bR film is self-assembled, undergoes controllable and reversible bending when the light-driven proton pump protein, bR, on the microcantilever surface is activated by visible light. Several control experiments are carried out to preclude the influence of heat and photothermal effects. It is shown that the nanomechanical motion is induced by the resulting gradient of protons, which are transported from the $\mathrm{KCl}$ solution on the cytoplasmic side of the bR film towards the extracellular side of the $b R$ film. Along with a simple physical interpretation, the microfabricated cantilever interfaced with the organized molecular film of bR can simulate the natural machinery in converting solar energy to mechanical energy.
\end{abstract}

(Some figures in this article are in colour only in the electronic version)

\section{Introduction}

The integration of molecular biology and the physical sciences at micro- and nanoscales for constructing hybrid devices has undergone rapid development [1-5]. Important requirements for the construction of hybrid devices include

(a) a suitable structure to act as a physical transducer for quantitative detection,

(b) special biomaterials and molecules for converting chemical energy into mechanical energy, and

(c) the implementation of interfacing technologies to combine the special biomaterials and molecules with the transducer.

Some studies have been carried out to meet the requirements mentioned above [6-10]. For the physical transducer, it is possible to use a microcantilever with biomaterials and biomolecules to construct a hybrid device. The microcantilever is selected because of its simple structure and good mechanical properties. It has already

\footnotetext{
3 Author to whom any correspondence should be addressed.
}

been applied in the development of biosensors [10-14] and bioactuators [4]. For a microcantilever used in a bioactuator, some biomaterials and biomolecules, which are immobilized on the microcantilever surface, are used to convert chemical energy into mechanical energy. Various functional materials and molecules have been developed for constructing hybrid devices $[6,7,15,16]$. In those materials, bacteriorhodopsin $(\mathrm{bR})$ has attracted particular interest due to its unique photochemical and photophysical properties. Bacteriorhodopsin (bR) is a photosensitive protein complex in the purple membrane isolated from Halobacterium salimarum, and may function as a light-driven proton pump [17, 18], which is capable of transporting protons across the membrane in a cell. The protein is formed from seven $\alpha$ helical transmembrane segments, and contains an all-trans retinal chromophore, covalently bound to the Lys-216 of the protein as a protonated Schiff base [19]. In the presence of visible light, bR goes through a photocycle involving a sequence of intermediate states (I, J, K, L, M, N, and O), during which a proton is pumped from the cytoplasmic side to the extracellular side, and a transmembrane proton gradient is 
generated [20]. During the photocycle, the proton on the protonated Schiff base, which is responsible for the absorption of light at a maximum of approximately $570 \mathrm{~nm}$, is transferred to the negatively charged Asp-85 located in the extracellular region [21, 22], and subsequently a proton from other amino acid residues, Glu-194 and Glu-204, is released into the solution on the extracellular side of the memebrane [23-25]. The protonated Schiff base, in turn, is reprotonated from the cytoplasmic solution by the initial Asp-96 located at the cytoplasmic region [26, 27]. The bR membrane protein, besides its unique photochemical properties [28], also has extraordinary stability, tolerating fairly broad $\mathrm{pH}$ and temperature ranges. It is, therefore, one of the most promising biomaterials for energy conversion, and there are already a broad range of applications in optoelectronics [29], optoelectronic sensors [30, 31], optical switches [32], optical storage [33], and information processing [34]. In bR-based applications, because its performance is greatly dependent on how the nonrandomly oriented $b R$ film is assembled on the solid surface, it is important that highly oriented bR film should be immobilized on the solid surface to generate a highly efficient electric response [35]. A number of techniques, such as Langmuir-Blodgett (LB) deposition [36], electrical field sedimentation (EFS) [37], electrostatic layer-by-layer (LBL) adsorption [38, 39], antigen-antibody molecular recognition [40], immobilizing matrixes [41, 42], and solgel encapsulation [43], have been developed to fabricate bR films on solid surfaces. These methods usually produce lowly oriented bR films, which cannot sustain high biological activity when immobilized on solid supports. The study of well defined inorganic surfaces and organic species now takes an increasing interest in self-assembly techniques.

In this paper, a microcantilever interfaced with bR film is used to construct a molecular machine-based bioactuator that allows reversible motion through switching visible light on and off. In order to construct the nanomechanical hybrid device by combining a microcantilever with $\mathrm{bR}$ in the purple membrane, a step-by-step adsorption technique based on biotin-streptavidin linkage is used to spatially control the arrangement and assembly of the purple membrane on the microcantilever surface. This technique can provide highly oriented bR film on the microcantilever surface, which can sustain high biological activity in the hybrid device. In order to demonstrate the performance of the nanomechanical device based on the light-driven pump, different experiments are carried out to assemble the bR film on the gold surface and the $\mathrm{Si}_{3} \mathrm{~N}_{4}$ surface of the microcantilever, respectively. When illuminated by visible light, the microcantilever generates a nanomechanical deflection response. In addition, several control experiments are carried out to preclude the influence of heat and photothermal effects. We demonstrate that the nanomechanical motion is caused by the gradient of protons transported from the solution towards the interface between the microcantilever surface and the bR film.

\section{Experimental details}

\subsection{Materials for constructing the hybrid device}

Streptavidin, NHS-biotin and cysteamine (CE) were purchased from Sigma. 6-((6-((biotinoyl)amino)hexanoyl)amino) hexanoic acid, sulfosuccinimidyl ester and sodium salt (biotinXX SSE) were purchased from Molecular Probes. 11-Amino1-undecanethiol and hydrochloride were purchased from Dojindo. APTES (3-aminopropyltriethoxysilane) was purchased from Aldrich Chemical Co. Other analytical reagents were purchased from Fluka. Double-distilled water, absolute ethanol and dimethyl formamide (DMF) were deoxygenated under argon before use. Commercially available V-shaped microcantilevers and rectangular microcantilevers, made of silicon nitride $\left(\mathrm{Si}_{3} \mathrm{~N}_{4}\right)$, were used in the experiments. The material's Young's modulus was $E=1.6 \times 10^{11} \mathrm{~N} \mathrm{~m}^{-2}$. The V-shaped microcantilevers were $200 \mu \mathrm{m}$ in length, $0.5 \mu \mathrm{m}$ in thickness and $20 \mu \mathrm{m}$ for each leg (Digital Instruments, CA). The rectangular microcantilevers were $200 \mu \mathrm{m}$ in length, $0.5 \mu \mathrm{m}$ in thickness and $20 \mu \mathrm{m}$ in width (Digital Instruments, CA).

\subsection{Preparation of bacteriorhodopsin and labelling of purple membrane by biotin}

The purple membrane was prepared through standard procedures, and kept in distilled water at $4{ }^{\circ} \mathrm{C}$ [44]. The biotin labelling procedure was carried out in our laboratory in the following way [35, 45]. First, $1 \mathrm{ml}$ of $4 \mathrm{mg} \mathrm{ml}^{-1}$ suspension of the purple membrane was biotinylated by incubation in a $10 \mathrm{mg} \mathrm{ml}^{-1}$ freshly prepared solution of biotin-XX SSE in a $1 \mathrm{M}$ sodium bicarbonate solution $(\mathrm{pH}$ 8.5). Next, after being stirred for $1 \mathrm{~h}$, the sample was washed three times by centrifugation, and resuspended in $0.1 \mathrm{M}$ sodium bicarbonate solution ( $\mathrm{pH} 8.5)$, and left overnight to remove unwanted biotin. Finally, the sample was dialyzed against $10 \mathrm{mM}$ PBS buffer $\left(2.7 \mathrm{mM} \mathrm{NaH} \mathrm{PO}_{4}, 7.3 \mathrm{mM} \mathrm{Na} \mathrm{HPO}_{4}, 150 \mathrm{mM} \mathrm{NaCl}\right.$, $\mathrm{pH}$ 7.2) for 2 days at $4{ }^{\circ} \mathrm{C}$.

\subsection{Assembling purple membrane on the gold surface of microcantilever}

Highly stable molecular layers prepared by the step-bystep self-assembly method were used for developing hybrid devices [46, 47] as illustrated schematically in figure 1(a). First, the commercially available microcantilever was cleaned using piranha solution (a mixture of $3: 7 \mathrm{v} / \mathrm{v}$ of $30 \% \mathrm{H}_{2} \mathrm{O}_{2}$ and $98 \% \mathrm{H}_{2} \mathrm{SO}_{4}$ ) for $25 \mathrm{~min}$, and rinsed with double distilled water three times for $5 \mathrm{~min}$, followed by drying under clean air. Then, the clean microcantilever was immersed into a 0.1 $\mathrm{M}$ solution of cysteamine (CE) in $10 \mathrm{ml}$ of ethanol under argon at room temperature for $24 \mathrm{~h}$, then rinsed with ethanol, and finally dried under clean air. Aminoalkanethiols, including cysteamine (CE) and 11-amino-1-undecanethiol, were used to modify the gold surface by introducing amino groups to it. The amino groups can usually be modified with amine-reactive biomaterials to functionalize the gold surface. Second, the microcantilever was immersed into a $0.01 \mathrm{M}$ solution of NHSbiotin in DMF $(10 \mathrm{ml})$ under argon, and allowed to react for $24 \mathrm{~h}$ at room temperature. The microcantilever was thoroughly rinsed with DMF, ethanol, and finally dried under clean air. Following this process, a stable biotin labelled microcantilever was obtained. Third, the microcantilever was immersed into a $0.05 \mathrm{mg} \mathrm{ml}^{-1}$ solution of streptavidin in PBS buffer $(2.7 \mathrm{mM}$ $\mathrm{NaH}_{2} \mathrm{PO}_{4} 7.3 \mathrm{mM} \mathrm{Na} \mathrm{HPO}_{4}, 150 \mathrm{mM} \mathrm{NaCl}, \mathrm{pH}$ 7.2), then rinsed with $\mathrm{PBS}$ buffer to remove the free streptavidin. Finally, the biotinylated purple membrane was assembled on the gold surface of the microcantilever by biotin-streptavidin linkage. 


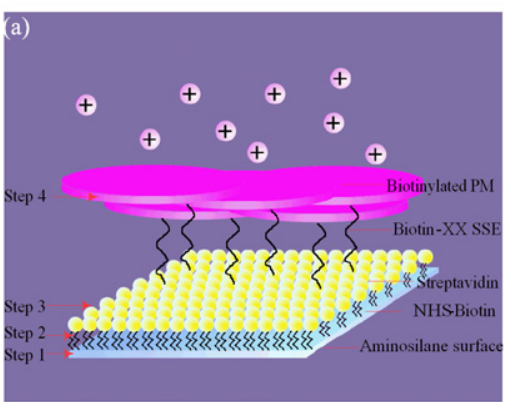

Optical Input

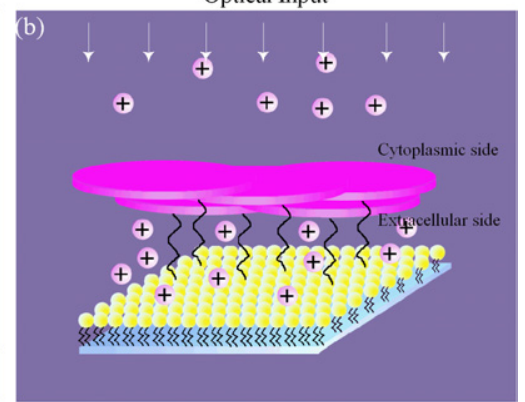

Optical Input
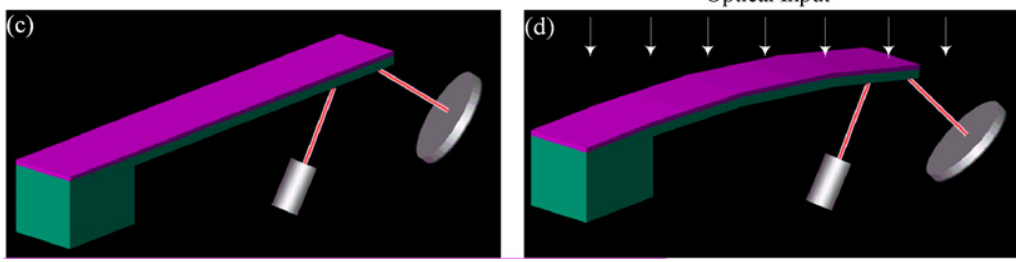

Figure 1. Schematic illustration of assembling the purple membrane on the microcantilever surface and the experimental system. (a) Procedures for assembling the purple membrane on the solid surface. (b) Illuminated by visible light, bR pumps the proton across the membrane to the interface between the microcantilever surface and the purple membrane. (c) The optical beam deflection is used to measure the deflection of the microcantilever. (d) Illuminated by visible light, the beam bends away from the purple membrane side.

\subsection{Assembling purple membrane on the $\mathrm{Si}_{3} \mathrm{~N}_{4}$ surface of the microcantilever}

Biotinylation of the $\mathrm{Si}_{3} \mathrm{~N}_{4}$ surface of the microcantilever was accomplished by first functionalizing the microcantilever with a $1.0 \% \mathrm{v} / \mathrm{v}$ APTES (3-aminopropyltriethoxysilane) in an ethanol solution for $2 \mathrm{~h}$ [13]. This procedure was used to modify the $\mathrm{Si}_{3} \mathrm{~N}_{4}$ surface to introduce amino groups to it. Then the microcantilever was rinsed thoroughly with ethanol to remove any unwanted material. The microcantilever was subsequently immersed in a $0.01 \mathrm{M}$ solution of NHS-biotin in DMF (10 ml) for $24 \mathrm{~h}$ under argon, followed by thorough rinsing in DMF, ethanol, and finally drying under clean air. Then the microcantilever was incubated in a $0.05 \mathrm{mg} \mathrm{ml}^{-1}$ of streptavidin in PBS buffer $\left(2.7 \mathrm{mM} \mathrm{NaH}{ }_{2} \mathrm{PO}_{4}, 7.3 \mathrm{mM}\right.$ $\mathrm{Na}_{2} \mathrm{HPO}_{4}, 150 \mathrm{mM} \mathrm{NaCl}, \mathrm{pH}$ 7.2) to fix the streptavidin matrices on the $\mathrm{Si}_{3} \mathrm{~N}_{4}$ surface of the microcantilever. Finally, the biotinylated purple membrane was assembled on the $\mathrm{Si}_{3} \mathrm{~N}_{4}$ surface.

\subsection{Measurement of the deflection of nanomechanical device}

Optical cantilever-deflection techniques, as schematically shown in figure 1(c), were used measure the nanomechanical motion of the hybrid device produced by the gradient of protons. When the visible light from the low power laser diode was focused on the microcantilever apex, the laser beam was reflected by the microcantilever surface, and collected by a position sensitive photodiode, where a four-stage preamplifier was mounted directly on the back of the position-sensitive detector (PSD) to transform the currents produced by the incident light spot into voltages. And a normalized voltage difference $\Delta V=\left(V_{1}-V_{2}\right)\left(V_{1}+V_{2}\right)^{-1}$ was digitized to an 8-bit value in an analog-to-digital converter, which determined the position of the reflected light spot. Then, the changes in the deflection were continuously monitored as well as stored on a computer. Such optical cantilever-deflection techniques were capable of measuring deflection with an accuracy of $0.1 \mathrm{~nm}$

\section{Results and discussion}

\subsection{Characterization of the biotinylated purple membrane}

To successfully construct a hybrid device by integrating a microcantilever with light-driven proton pumps, bR, it is important that the characterization of the biotinylated $\mathrm{bR}$ is measured before the purple membrane is assembled on the microcantilever. Figure 2(a) shows the UV-vis absorption spectra of the native purple membrane and the biotinylated purple membrane measured by the Hitachi U3200 spectrophotometer. It can be seen from this figure that the absorption ratio of $A_{280 \mathrm{~nm}} / A_{570 \mathrm{~nm}}$ is below 2.0, suggesting that it is a good quality sample, and the shift of the adsorption wavelength between the native purple membrane and the biotinylated purple membrane is less than $2 \mathrm{~nm}$, indicating that biotinylation of the purple membrane does not change the conformation of bR. Figure 2(b) shows the flash kinetic spectrum of the native purple membrane and the biotinylated purple membrane at $412 \mathrm{~nm}$. The slight change of the flash kinetic absorption reveals that biotinylation of the purple membrane does not affect its photochemical activity for further pumping protons. From the two figures, it can be seen that the biological activity of the biotinylated purple membrane is still sustained, and the biotinylated purple membrane can be used to construct the hybrid device. In order to demonstrate the assembly of the biotinylated purple membrane on the microcantilever surface, immobilization of the purple membrane on a solid surface was monitored in real time by surface plasmon resonance (SPR) under a fixed angle of incident light to record the changes in the refractive index against the reflected intensity. The surface mass density $\left(\mathrm{ng} \mathrm{mm}^{-2}\right)$ of the protein immobilized on the 

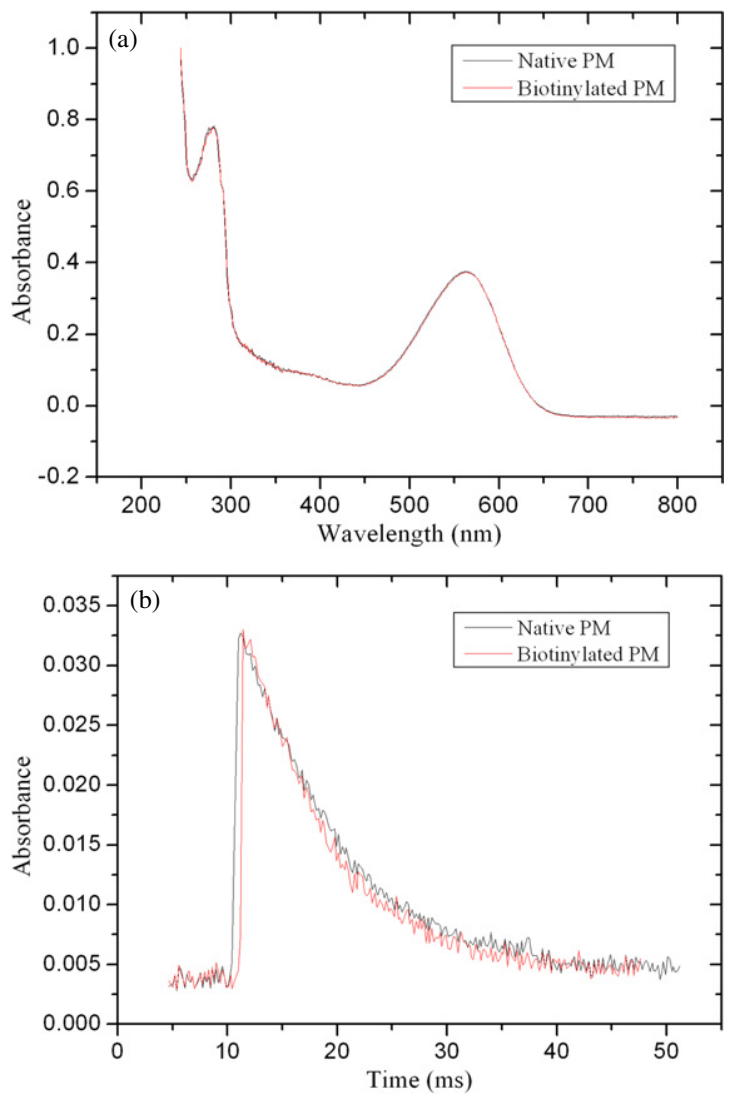

Figure 2. (a) UV-vis absorption spectra of the native purple membrane and the biotinylated purple membrane. The absorption ratio of $A_{280 \mathrm{~nm}} / A_{570 \mathrm{~nm}}$ is below 2.0, suggesting that it is a good quality sample, and the shift of the adsorption wavelength between the native purple membrane and the biotinylated purple membrane is less than $2 \mathrm{~nm}$, indicating that biotinylation of the purple membrane does not change the conformation of $b R$. (b) Flash kinetic spectrum of the native purple membrane and the biotinylated purple membrane at $412 \mathrm{~nm}$. The slight change of the flash kinetic absorption reveals that biotinylation of the purple membrane does not affect its biological activity.

surface varies linearly with the change in the resonance angle $\left(\Delta \Phi_{\mathrm{SPR}}\right)$, and a $0.1^{\circ}$ shift of $\Delta \Phi_{\mathrm{SPR}}$ represents $0.55 \mathrm{ng} \mathrm{mm}^{-2}$ of protein adsorbed. The number density of protein molecules can be calculated as $6.023 \times 10^{15} \times 0.55 \times$ $\Delta \Phi_{\mathrm{SPR}} / \mathrm{MW}$ (number $\mathrm{mm}^{-2}$ ) according to Stenberg et al [48], where MW is molecular weight. Using this method, the surface density of the purple membrane bound to the solid surface can be obtained. In our experiments, the purple membrane was assembled on the gold surface and $\mathrm{Si}_{3} \mathrm{~N}_{4}$ surface of the microcantilever, respectively. If the purple membrane was assembled on the gold surface, 11-amino-1-undecanethiol and cysteamine (CE) were used to form the aminosilane surface. They have a similar structure, with a thiol group $(-\mathrm{SH})$ on one end and an aminosilane group $\left(-\mathrm{NH}_{2}\right)$ on the other, as given by $\mathrm{SH}-\left(\mathrm{CH}_{2}\right)_{n}-\mathrm{NH}_{2}$, which represents 11-amino1-undecanethiol when $n=11$, and cysteamine (CE) when $n=2$. The thiol group can be tightly bound to the gold surface of the microcantilever surface, and the aminosilane group can be used to link NHS-biotin in the next step. If the purple membrane was assembled on the $\mathrm{Si}_{3} \mathrm{~N}_{4}$ surface,

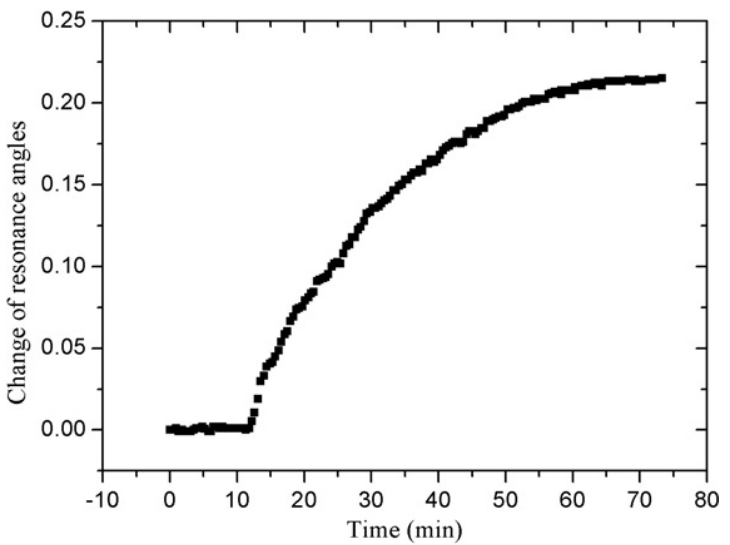

Figure 3. SPR measurement of the change in the resonance angles during assembly of the purple membrane on the microcantilever surface.

APTES (3-aminopropyltriethoxysilane) was first assembled on the $\mathrm{Si}_{3} \mathrm{~N}_{4}$ surface to form the aminosilane surface. APTES (3-aminopropyltriethoxysilane) can be directly formed on the $\mathrm{Si}_{3} \mathrm{~N}_{4}$ surface, and provides the aminosilane group on the $\mathrm{Si}_{3} \mathrm{~N}_{4}$ surface for binding NHS-biotin in the next step. After the gold or $\mathrm{Si}_{3} \mathrm{~N}_{4}$ surface has been modified by 11-amino1-undecanethiol/cysteamine and APTES, respectively, NHSbiotin can be assembled on the aminosilane surface. Then, streptavidin can be bound to specific regions of the biotinylated surface by the chemical linkage between NHS-biotin and streptavidin. Finally, the biotinylated bR film can be assembled on the microcantilever surface by the biotin-streptavidin linkage. Here, we tested the immobilization of the purple membrane on the gold surface. After the CE monolayer, NHSbiotin and streptavidin were assembled on the gold surface with a gold layer (about $30 \mathrm{~nm}$ ), respectively, the resonance angles were measured by SPR. Figure 3 shows the change in resonance angle when the biotinylated purple membrane was assembled on the gold surface by biotin-streptavidin linkage. From this figure, the surface density of protein bound to the surface can be calculated as about $10^{10}-10^{11} \mathrm{~mm}^{-2}$ according to Stenberg et al [48]. This density is high, and consistent with that in [35]. It is demonstrated that the bR film was densely formed on the solid support by the step-by-step self-assembly method in our experiments. The experimental condition is the same as that for assembling the purple membrane on the microcantilever. Figures 4(a) and (b) show a SEM micrograph of the solid surface when the surface is inert and the purple membrane is not assembled on it, and that of the biotinylated purple membrane assembled on the solid surface with the streptavidin layer, respectively. Compared with figures 4(a) and (b), it can be seen that the biotinylated purple membrane is formed densely on the streptavidin layer based on the biotinstreptavidin linkage, and is not assembled on the inert solid surface without chemical linkage. And the overlap of the segments of the membrane is observed in figure 4(b), which shows that the labelled bR film is arrayed densely on the streptavidin layer on the solid surface. Finally (see figures 3 and 4), the biotinylated bR film is assembled on the solid surface based on the biotin-streptavidin linkage by the stepby-step self-assembly method. This self-assembly method is effective for the fabrication of a bio-optical device. 

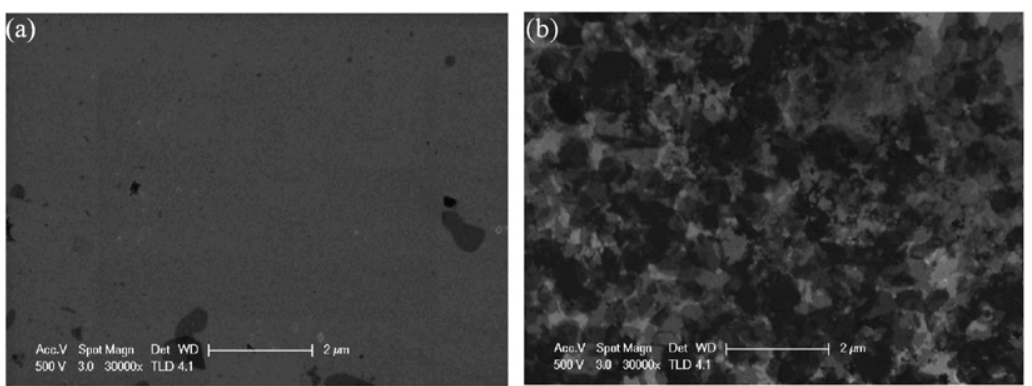

Figure 4. (a) SEM micrograph of the solid surface when the surface is inert, and the purple membrane was not assembled on it; (b) SEM picture of the biotinylated purple membrane assembled on the streptavidin layer on the solid surface.

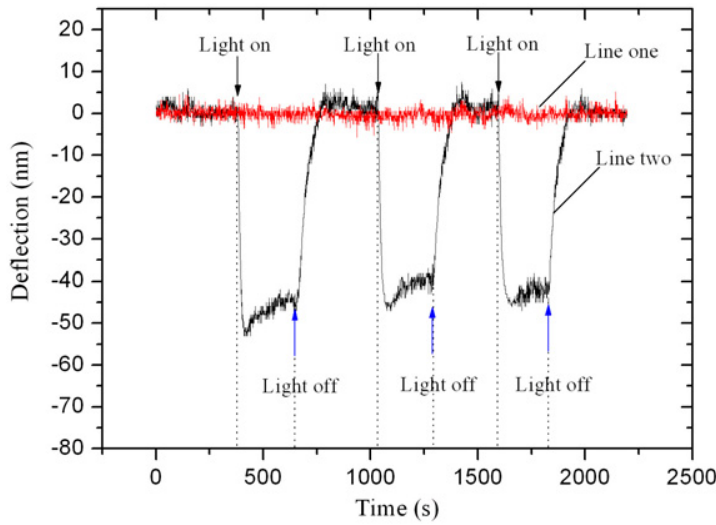

Figure 5. Deflection of the microcantilever as a function of time with switching the visible light on and off when the purple membrane was assembled on the gold surface of the microcantilever. Line one represents the deflection of the microcantilever in the control experiment, in which the gold surface of the microcantilever was inert, and the purple membrane was not assembled on it.

\subsection{Properties of the hybrid device}

In our experiment, after the biotinylated purple membrane was assembled on the microcantilever surface as shown in figure 1(a), the microcantilever was mounted into the head of an atomic force microscope (SPM6800). Once the microcantilever was immersed in the electrolyte buffer (100 $\mu \mathrm{mol} \mathrm{l}^{-1} \mathrm{KCl}, 5 \mu \mathrm{mol}^{-1} \mathrm{CaCl}_{2}$, pH 6.8), the deflection was continuously monitored in real time, and the data were stored on a computer. Before each experiment, the microcantilever was equilibrated in the electrolyte buffer to decrease the drift effect until a stable baseline of deflection was obtained. Then, the visible light was turned on to illuminate the purple membrane on the microcantilever surface. The visible light can cause a conformational change in $\mathrm{bR}$, which pumps the protons in solution to the interface between the microcantilever and the purple membrane as schematically shown in figure 1(b). Finally, a high gradient of protons will cause the microcantilever to bend away from the purple membrane side as shown in figure 1(d). Figure 5 shows the deflection profile of the microcantilever as a function of time with switching the visible light on and off. In this figure, line two denotes the deflection response of the microcantilever when the biotinylated purple membrane assembled on the gold surface of the microcantilever is activated by the visible light.
In order to confirm that the beam bending was not due to heat or photothermal effects, control experiments were necessary. When the microcantilever surface was illuminated, only an extremely small amount of light energy could be converted into heat energy to bend the microcantilever. The deflection of the microcantilever response to a uniform light energy was estimated to be $0.05 \mathrm{~nm}$ when the input optical power was $1 \mathrm{nW}$ [49]. The thermal conductivity of the self-assembled membrane is not known, however, it is as small as that of silicon dioxide [50]. And the membrane is extremely thin with a thickness of about $5 \mathrm{~nm}$. Thus, the deflection of the microcantilever can be estimated to be lower than $3.6 \mathrm{~nm}$ when a $1 \mathrm{~mW} \mathrm{~cm}^{2}$ visible light illuminates the $\mathrm{bR}$ on the microcantilever according to $[49,51]$. In addition, in our experiment, the microcantilever was immersed in a liquid cell, which was mounted on a thermoelectric cooler so that the liquid in the cell was always in a thermal equilibrium. Thus, thermal effects due to the visible light can be ignored. Line one in the figure denotes the deflection of the microcantilever in the control experiment, in which the microcantilever surface is inert and the purple membrane is not assembled on the microcantilever surface. From the line, there is no deflection change when the surface is inert. This shows that the heat and photothermal effects of visible light do not contribute to the change in the deflection response, and the origin of the nanomechanical deflection is generated by the protons pumped by the purple membrane from the electrolyte buffer to the interface between the microcantilever surface and the purple membrane. Furthermore, in order to demonstrate that the nanomechanical deflection is due to $\mathrm{bR}$ pumping the protons to the cantilever interface, another experiment is carried out, in which the purple membrane is assembled on the $\mathrm{Si}_{3} \mathrm{~N}_{4}$ surface of the microcantilever. Using the same experimental conditions, switching the visible light on and off, the deflection response of the microcantilever is observed as shown in figure 6. And a control experiment, in which the purple membrane is not assembled on the $\mathrm{Si}_{3} \mathrm{~N}_{4}$ surface, is carried out to demonstrate that the deflection is due to the high gradient of protons pumped by bR. From figures 5 and 6 , the observed deflection peak when first switching on the visible light increases to about $45 \mathrm{~nm}$ after about several tens of seconds. Then, the deflection slowly decreases until another equilibrated deflection is obtained. The peak deflection corresponds to the peak concentration of the protons pumped by bR. The difference between the peak deflection and the deflection when the microcantilever is equilibrated again is about $10 \mathrm{~nm}$. 


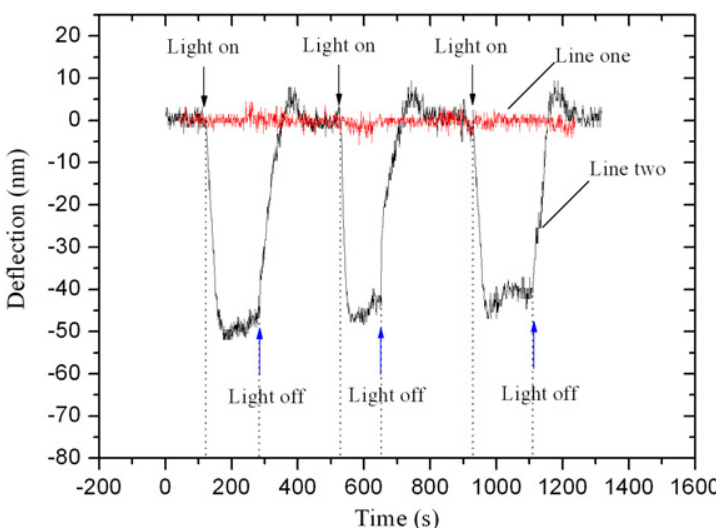

Figure 6. Deflection of the microcantilever as a function of time with switching the visible light on and off, when the purple membrane was assembled on the $\mathrm{Si}_{3} \mathrm{~N}_{4}$ surface of the microcantilever. Line one represents the deflection of the microcantilever in the control experiment, in which the $\mathrm{Si}_{3} \mathrm{~N}_{4}$ surface of the microcantilever was inert, and the purple membrane was not assembled on it.

Continuously switching the visible light on and off decreases the peak deflections. The reason for this is that continuous visible light can bleach the purple membrane, decreasing its biological activity over time [52]. From the results, the deflection of the nanomechanical device is generated by the high gradient of protons, and is not related to whether the purple membrane is assembled on the gold surface or the $\mathrm{Si}_{3} \mathrm{~N}_{4}$ surface of the microcantilever. In fact, the response of the microcantilever generated by the gradient of protons can be considered as a redox reaction on the microcantilever surface. Its mechanism is the same as that of photo-driven mechanical cyclic motion [53] and that of the redox-driven mechanical switching in providing mechanical motion of the microcantilever when the linear motor, bistable rotaxanes, selfassembled on the microcantilever surface [4] are exposed to the aqueous oxidant and reductant solution. From figure 5, the response timescale is about $40 \mathrm{~s}$ from switching the light on to the peak deflection, and the velocity of the deflection response is less than $2 \mathrm{~nm} \mathrm{~s}^{-1}$. This response time is of the same order as the response timescale of $\sim 60 \mathrm{~s}$ in [4], and lower than that in [53]. Moreover, the response time is also of the same order as that of ssDNA assembly on microcantilevers $[10,12,13]$ A plausible explanation for the slow response is that the protons pumped by bR must be rearranged in the interface before saturation. Furthermore, because the dimension of the microcantilever is in micrometres, the Reynolds number is extremely small, therefore the deflection response can be considered a static one. Then, the small change in the surface stress may drive the microcantilever in a slow stress process. Here, a simple model of deflection relating to the surface stress can be used to explain the experiments. The change in surface stress between the upper and lower surfaces of the microcantilever is deduced from the measured deflection using Stoney's formula [54]

$$
\Delta \sigma=\frac{E t^{2}}{6 R(1-v)},
$$

where $R^{-1}=3 \Delta w /\left(2 L^{2}\right)$ is the curvature of the microcantilever, $\Delta w$ is the deflection of the free end, $L$ is the length, $t$ is the thickness, $E$ is Young's modulus, and $v$ is Poisson's ratio of the microcantilever material. From figures 5 and 6 , a mean value of surface stress, $15 \mathrm{mN} \mathrm{m}^{-1}$, is obtained for a $100 \mu \mathrm{M} \mathrm{KCl}$ solution ( $\mathrm{pH}$ 6.8). This equation relates the change in surface stress to the change in deflection, and does not give a direct physical interpretation of the deflection, which might be complicated. From figures 5 and 6 , when the bR film assembled on the gold or $\mathrm{Si}_{3} \mathrm{~N}_{4}$ surface is activated by the visible light, the protons are transported from the $\mathrm{KCl}$ solution into the interface between the bR film and the gold surface or the $\mathrm{Si}_{3} \mathrm{~N}_{4}$ surface of the microcantilever. And the protons induce a change in the electric double layer on the microcantilever. Then, the strong positive charge of the protons in the electric double layer tend to repel the protons from each other, and cause the extension of the top surface of the microcantilever. Thus, the microcantilever is bent away from the purple membrane side as shown in figure 1, which is bent downward. Here, the microcantilever interfaced with the purple membrane can be considered as an asymmetric membrane. The curvature of the asymmetric membrane varies with the electrical potential difference across the purple membrane due to the electric double layer [55]. Then, the deflection can be directly related to the electrical potential difference across the purple membrane, $\Delta w \propto \Delta \varphi[55,56]$. The electrical potential difference across the purple membrane is determined by the ionic strength according to the theory of the electric double layer (EDL) [57, 58], and the ion concentration of the electrolyte buffers plays a prominent role in the deflection of the microcantilever [55]. The energy conversion efficiency can be expressed as

$$
\eta=\frac{E_{\mathrm{mech}}}{E_{\mathrm{opt}}}
$$

where $E_{\text {mech }}=\frac{1}{2} k \cdot \Delta z^{2}$ and $E_{\text {opt }}=A_{\text {cant }} \cdot \Psi$ are the mechanical energy of the microcantilever and the optical energy, respectively, $k=0.12 \mathrm{~N} \mathrm{~m}^{-1}$ is the effective spring constant of the microcantilever, $\Delta z$ is the deflection at the free end of the microcantilever, $A_{\text {cant }}$ is the surface area of the microcantilever, and $\Psi$ is the light intensity. The mechanical energy can be calculated as approximately $1.5 \times 10^{-16} \mathrm{~J}$ for a $50 \mathrm{~nm}$ deflection of the microcantilever used in our experiments. And the optical energy is estimated to be about $3.2 \times 10^{-8} \mathrm{~J}$ when the light with an intensity of $0.1 \mathrm{~mW} \mathrm{~cm} \mathrm{~cm}^{-2}$ calibrated by the laser powermeter (T148) illuminates the bR film on the microcantilever surface, whose dimensions are $200 \mu \mathrm{m}$ in length, and $20 \mu \mathrm{m}$ in width for each leg. Thus, the energy conversion efficiency according to equation (2) in our experiments is estimated to be about $0.0000005 \%$. This value is slightly lower than the energy conversion efficiency of $0.000001 \%$ calculated by equation (2) when the light with an intensity about $6.5 \mathrm{~mW} \mathrm{~cm}^{-2}$ illuminates the microcantilever modified by a monolayer of azobenzene molecules in [53], whose dimensions are $180 \mu \mathrm{m}$ in length, $40 \mu \mathrm{m}$ in leg width, and $2 \mu \mathrm{m}$ in thickness. Figure 7 shows the deflection profiles of the hybrid device as a function of time when different concentrations of electrolyte buffer are used. Figure 8 shows the deflection as a function of concentration of the electrolyte buffer. From the two figures, it can be seen that the deflection is decreased with increasing electrolyte buffer concentration. And the deflection of the 


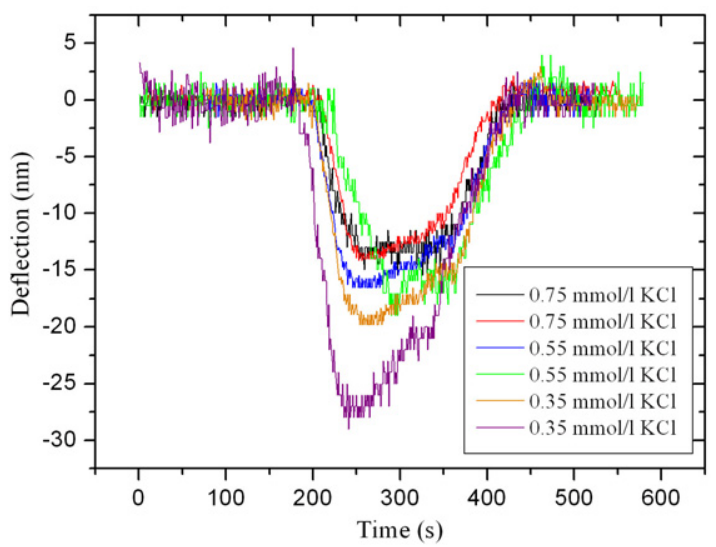

Figure 7. Deflection profiles of the hybrid device as a function of time when different concentrations of electrolyte buffers were used.

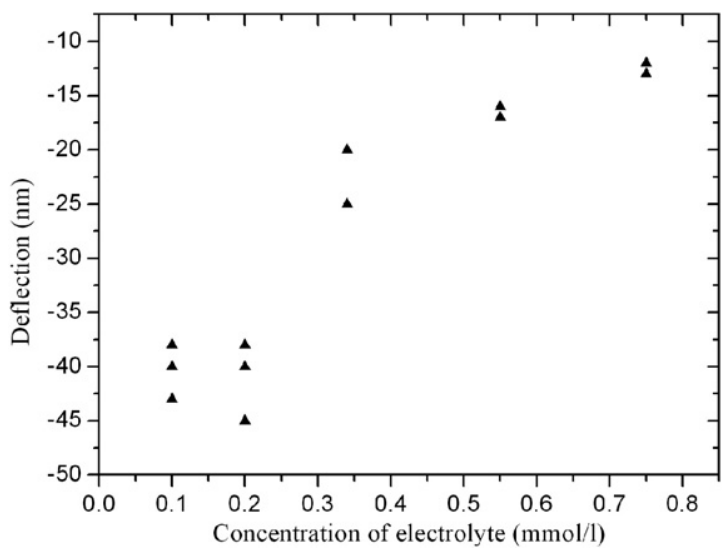

Figure 8. Deflections of the microcantilever with respect to the different concentrations of the electrolyte buffer.

microcantilever depends nonlinearly on the concentration of the electrolyte. The reason for this is that the electrical potential difference across the purple membrane depends on the Debye length, which relates nonlinearly to the electrical potential difference $[57,58]$. In summary, the deflection of the device is generated by the electrical potential contribution from the proton. The ion concentration in the electrolyte buffer can affect the surface electrical potential and, in turn, affect the deflection of the microcantilever. So we can control the direction of motion by switching the visible light on and off, and control the magnitude of the motion of the nanomechanical device by changing the ionic strength of the electrolyte buffer.

\section{Conclusions}

Bacteriorhodopsin (bR) is one of the most promising biomaterials for energy conversion, and can be used to construct bR-based devices. In this work, a layer-by-layer method based on the self-assembly technique is used to assemble biotinylated purple membrane on the gold surface and the $\mathrm{Si}_{3} \mathrm{~N}_{4}$ surface of a microcantilever, respectively, to construct a hybrid device. When switching the visible light on and off, the microcantilever coated with the purple membrane undergoes controllable and reversible bending. From the control experiments, it can be seen that the nanomechanical deflection is caused by the electric potential induced by the protons transported by bR from the electrolyte buffer to the interface between the microcantilever surface and the purple membrane. Therefore, by integrating MEMS/NEMS with a nano-biomolecular machine, bR, the molecular machine-based bioactuator can simulate the natural machinery in directly converting solar energy into mechanical energy. This will impact many areas, and offer new potential applications in the future.

\section{Acknowledgments}

This research was supported by the National Natural Science Foundation of China (NSFC) (grant Nos 10225209 and 90305020), key project from the Chinese Academy of Sciences (grant No. KJCX2-SW-L2).

\section{References}

[1] Teoh Y P, Dunn G M, Mackenzie I and Priestley N 2004 Semicond. Sci. Technol. 19885

[2] Reich D H, Tanase M, Hultgren A, Bauer L A, Chen C S and Meyer G J 2003 J. Appl. Phys. 937275

[3] Soong R K, Bachand G D, Neves H P, Olkhovets A G, Craighead H G and Montemagno C D 2000 Science 2901555

[4] Huang T J, Brough B, Ho C M, Li Y, Flood A H, Bonvallet P A, Tseng H R, Stoddart J F, Baller M and Magonov S 2004 Appl. Phys. Lett. 855391

[5] Sepaniak M J, Datskos P G and Lavrik N V 2002 Anal. Chem. $74668 \mathrm{~A}$

[6] Baughman R H et al 1999 Science 2841340

[7] Schreiber F 2001 J. Phys.: Condens. Matter 16881

[8] Noji H, Yasuda R, Yoshida M and Kinosita K J 1997 Nature 386299

[9] Salem A K, Chao J, Leong K W and Searson P C 2004 Adv. Mater. 16268

[10] Fritz J, Baller M K, Lang H P, Rothuizen H, Vettiger P, Meyer E, Güntherodt H-J, Gerber C and Gimzewski J K 2000 Science $\mathbf{2 8 8} 316$

[11] Wu G, Datar R H, Hansen K M, Thundat T, Cote R J and Majumdar A 2001 Nat. Biotechnol. 19856

[12] Hansen K M, Ji H, Wu G, Datar R H, Cote R J, Majumdar A and Thundat T 2001 Anal. Chem. 731567

[13] Wu G, Ji H, Hansen K M, Thundat T, Datar R H, Cote R J, Hagan M F, Chakraborty A K and Majumdar A 2001 Proc. Natl Acad. Sci. 98(4) 1560

[14] Mckendry R et al 2002 Proc. Natl Acad. Sci. 99(15) 9783

[15] Lanyi Y 1993 Biochim. Biophys. Acta 1183241

[16] Juodkazis S, Mukai N, Wakaki R, Yamaguchi A, Matsuo S and Misawa H 2000 Nature 408178

[17] Oesterhelt D and Stoeckenius W 1971 Nat. New Biol. 233149

[18] Blaurock A E and Stoeckenius W 1971 Nat. New Biol. 233152

[19] Rothschild K J, Argade P V, Earnest T N, Huang K S, London E, Liao M J, Bayley H, Khorana H G and Herzfeld J 1982 J. Biol. Chem. 2578592

[20] Osterhelt D and Stöckenius W 1973 Proc. Natl Acad. Sci. 70 2853

[21] Otto H, Marti T and Holz M 1990 Proc. Natl Acad. Sci. 86 9228

[22] Lanyi J K 1998 J. Struct. Biol. 124164

[23] Lazarova T, Sanz C, Querol E and Padros E 2000 Biophys. J. 782022

[24] Richter H-T, Brown L S, Needleman R and Lanyi J K 1996 Biochemistry 354054

[25] Brown L S, Sasaki J, Kandori H, Maeda A, Needleman R and Lanyi J K 1995 J. Biol. Chem. 27027122 
[26] Dioumaev A K, Brown L S, Needleman R and Lanyi J K 2001 Biochemistry $\mathbf{4 0} 1130$

[27] Miller A and Oesterhelt D 1990 Biochim. Biophys. Acta 1020 57

[28] Druzhko A B, Robertson B, Alvarez R, Lera A R and Weetall H H 1998 Biochim. Biophys. Acta 1371371

[29] Singh C P and Roy S 2003 Curr. Appl. Phys. 3163

[30] Lensu L, Parkkinen J, Parkkinen S, Frydrych M and Jaaskelainen T 2003 Opt. Mater. 21783

[31] Li Q, Stuart J A, Birge R R, Xu J, Stickrath A and Bhattacharya P 2004 Biosens. Bioelectron. 1986

[32] Roy S, Sharma P, Dharmadhikari A K and Mathur D 2004 Opt. Commun. 237251

[33] Stuart J A, Marcy D L, Wise K J and Birge R R 2002 Synth. Met. 1273

[34] Min J, Choi H-G, Oh B-K, Lee W H, Paek S-H and Choi J-W 2001 Biosens. Bioelectron. 16917

[35] Chen D L, Lu Y J, Sui S F, Xu B and Hu K S 2003 J. Phys. Chem. B 1073598

[36] Chi L F, Anders M, Fuchs H, Johnston R R and Ringsdorf H 1993 Science 259213

[37] Kononenko A A, Lukashev E P and Maximychev A V 1986 Biochim. Biophys. Acta 850162

[38] He J A, Samuelson L, Li L, Kumar J and Tripathy S K 1998 Langmuir 141674

[39] Chu J F, Li X C, Zhang J P and Tang J 2003 Biochem. Biophys. Res. Commun. 305116

[40] Koyama K, Yamaguchi N and Miyasaka T 1994 Science 265 762

[41] Eklund K, Vuorinen J, Mikkola J, Virtanen J and Kinnunen P 1988 Biochemistry 273433
[42] Chen Z, Lewis A, Takei H and Nebenzahl I 1991 Appl. Opt. 30 5188

[43] Wu S G, Ellerby L M, Cohan J S, Dunn B, El-Sayed M A, Valentine J S and Zink J I 1993 Chem. Mater. 5115

[44] Osterhelt D and Stoeelcenius W 1974 Methods Enzymol. 31 667

[45] Henderson R, Jubb J S and Whytock S 1978 J. Mol. Biol. 123259

[46] Müller W, Ringsdorf H, Rump E, Wildburg G, Zhang X, Angermaier L, Knoll W, Liley M and Spinke J 1993 Science 2621706

[47] Yam C-M, Pradier C-M, Salmain M, Marcus P and Jaouen G 2001 J. Colloid Interface Sci. 235183

[48] Stenberg E, Pesson B, Roos H and Urbaniczky C $1991 \mathrm{~J}$. Colloid Interface Sci. 143513

[49] Gimzewski J K, Gerber Ch, Meyer E and Schlittler R R 1994 Chem. Phys. Lett. 217589

[50] Marie R, Jensenius H, Thaysen J, Christensen C B and Boisen A 2002 Ultramicroscopy 9129

[51] Datskos P G, Rajic S and Datskou I 2000 Ultramicroscopy 8249

[52] Dancsházy Z, Tokaji Z and Dér A 1999 FEBS Lett. 450154

[53] Ji H-F, Feng Y, Xu X, Purushotham V, Thundat T and Brown G M 2004 Chem. Commun. 222532

[54] Stoney G G 1908 Proc. R. Soc. A 82172

[55] Liu F, Zhang Y and Ou-Yang Z C 2003 Biosens. Bioelectron. 18655

[56] Ou-Yang Z C, Liu S and Xie Y Z 1991 Mol. Cryst. Liq. Cryst. 204143

[57] Hunter R J 2004 Foundations of Colloid Science (Oxford: Oxford University Press)

[58] Misra S and Varanasi S 1989 Macromolecules 224173 